\title{
Enhanced covalent interface, crosslinked network and gas barrier property of functionalized graphene oxide/styrene-butadiene rubber composites triggered by thiol-ene click reaction
}

\author{
Long Zheng \\ Beijing University of Chemical Technology \\ Stephen Jerrams \\ Technological University Dublin, stephen.jerrams@tudublin.ie \\ Tian Su \\ Beijing University of Chemical Technology
}

See next page for additional authors

Follow this and additional works at: https://arrow.tudublin.ie/cerart

Part of the Materials Chemistry Commons, and the Polymer and Organic Materials Commons

\begin{abstract}
Recommended Citation
Long Zheng, Stephen Jerrams, Tian Su, Zongchao Xu, Liqun Zhang, Li Liu, Shipeng Wen, Enhanced covalent interface, crosslinked network and gas barrier property of functionalized graphene oxide/ styrene-butadiene rubber composites triggered by thiol-ene click reaction, Composites Part B: Engineering, Volume 197, 2020, 108186, ISSN 1359-8368, DOI: 10.1016/j.compositesb.2020.108186.
\end{abstract}

This Article is brought to you for free and open access by the Centre for Elastomer Research at ARROW@TU Dublin. It has been accepted for inclusion in Articles by an authorized administrator of ARROW@TU Dublin. For more information, please contact arrow.admin@tudublin.ie, aisling.coyne@tudublin.ie,gerard.connolly@tudublin.ie. Funder: National Key R\&D Program of China; National Natural Science Foundation of China (NSFC)

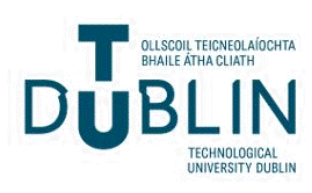




\section{Authors}

Long Zheng, Stephen Jerrams, Tian Su, Zongchao Xu, Liqun Zhang, Li Liu, and Shipeng Wen

This article is available at ARROW@TU Dublin: https://arrow.tudublin.ie/cerart/24 


\title{
Enhanced covalent interface, crosslinked network and gas barrier property of functionalized graphene oxide/styrene-butadiene rubber composites triggered by thiol-ene click reaction
}

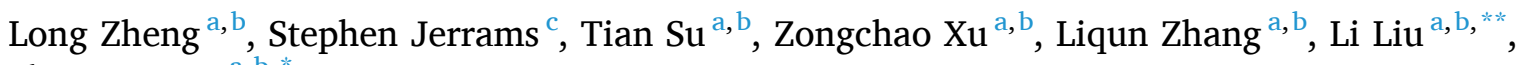 \\ Shipeng Wen ${ }^{\mathrm{a}, \mathrm{b}, *}$ \\ ${ }^{a}$ State Key Laboratory of Chemical Resource Engineering, Beijing University of Chemical Technology, Beijing, 100029, China \\ ${ }^{\mathrm{b}}$ Beijing Engineering Research Center of Advanced Elastomers, Beijing University of Chemical Technology, Beijing, 100029, China \\ ${ }^{\mathrm{c}}$ Centre for Elastomer Research, Technological University Dublin, Dublin, Ireland
}

\section{A R T I C L E I N F O}

\section{Keywords:}

Click reaction

Functionalized graphene oxide

Gas barrier properties

Rubber composites

\begin{abstract}
A B S T R A C T
The high gas barrier property of a rubber composite is of great significance for reducing the exhaust gas emissions due to tire rolling resistance and hence the contribution this factor makes to environmental protection. Enhanced covalent interfaces and crosslinked networks are crucial to the gas barrier property of rubber composites. In this research, $\gamma$-mercaptopropyltriethoxysilane (MPS) modified GO (MGO)/styrene-butadiene rubber (SBR) composites were prepared by a synergetic strategy of latex compounding method and thiol-ene click reaction. It was found that the mercapto groups in MGO reacted with the vinyl groups in SBR molecules through thiol-ene click reaction during the crosslinking process at $150{ }^{\circ} \mathrm{C}$, thus forming strong chemical interactions at the interface in the form of GO-MPS-rubber and enhanced crosslinked networks. Meanwhile, the strong interface promoted the dispersion of MGO in SBR. The uniform dispersion of MGO, strong interface between MGO and SBR molecules and enhanced crosslinked networks resulted in improved mechanical and gas barrier properties. When filling $5 \mathrm{phr}$ fillers, the tensile strength and gas barrier properties of an MGO/SBR composite improved by $19.0 \%$ and $37.5 \%$, respectively, relative to the comparing GO/SBR composite.
\end{abstract}

\section{Introduction}

In recent decades, the environmental pollution brought by vehicles, such as exhaust gas emissions, has attracted increasing attention [1]. In general, the low rolling resistance of tires is beneficial for decreasing exhaust gas emissions. Current studies for optimizing rolling resistance have mainly focused on the selection of raw materials for tire tread, rubber formulae and processing techniques [2-4]. However, the influence of the gas barrier property of tires on the rolling resistance has not received enough attention. According to a report by Walter $\mathrm{H}$. Waddell et al. [5], rolling resistance increased by $2.5 \%$ for every $10 \mathrm{kPa}$ loss in inflation pressure, thereby resulting increased exhaust gas emissions of vehicles. Therefore, preparation of rubber composites possessing high gas barrier property is of great significance for reducing exhaust gas emissions and consequently contributing to environmental protection.

As we known, most of the current tire inner liner layer are made of butyl rubber or halobutyl rubber due to its excellent gas barrier properties. However, butyl rubber has the disadvantages of poor processability and poor adhesion with carcass plies. Usually, using styrenebutadiene rubber (SBR) to replace or partially replace butyl rubber in the composition of tire inner liner layer is one of the ways to overcome these shortcomings [6,7]. But the gas barrier properties of SBR is relatively poor, it is necessary to improve the gas barrier properties of SBR through the combination with nanofillers. From a scientific perspective, construction of complex filler networks and crosslinked networks are critical to improving the gas barrier property of rubber composites. In previous studies, graphene oxide (GO) has been recognized as one of the ideal nanofillers to prepare high barrier rubber composites, owing to the single layered thickness and large surface area of GO [8,9]. However, owing to the abundant oxygen groups on GO sheets, the surface energy between hydrophilic GO sheets and hydrophobic rubber molecules is quite different [10]. This results in poor dispersion of GO and

\footnotetext{
* Corresponding author. State Key Laboratory of Chemical Resource Engineering, Beijing University of Chemical Technology, Beijing, 100029, China.

** Corresponding author. State Key Laboratory of Chemical Resource Engineering, Beijing University of Chemical Technology, Beijing, 100029, China.

E-mail addresses: liul@mail.buct.edu.cn (L. Liu), wensp@mail.buct.edu.cn (S. Wen).
} 
inadequate interface interactions between GO and rubber molecules, deteriorating the ultimate performance of the rubber products. Thus, the functionality of GO needs to be improved to tune its surface properties and tailor the interface chemistry between GO and rubber molecules.

In general, compatibility between modified GO and rubber molecules would be enhanced after the surface modification of GO. However, it's difficult to form sufficient interface interactions between modified GO with non-polar rubber molecules such as SBR. In fact, construction of chemical interfaces between fillers and rubber molecules in non-polar rubber composites is an effective method for preparing rubber composites with superior overall performance $[11,12]$. Therefore, designing the surface chemistry of GO and constructing chemical interfaces in rubber composites are conductive to improving the barrier property of SBR.

Recently, click chemistry has been widely used in the surface treatment of composites due to its mild reaction conditions and high efficiency [13-15]. In particular, a thiol-ene click reaction, which is one of the four categories of click reactions, can be easily triggered by UV light or heat and no metal catalyst is needed [16-18]. Its fast reaction speed, high yield and freedom from the effects of oxygen show greater advantages in the interfacial modification of rubber composites. Kanoth et al. [19]. grafted mercapto groups onto the surface of cellulose nanocrystals (CNCs) and the modified CNCs (m-CNCs) were filled into the natural rubber (NR) matrix by solution casting. A three-dimensional chemical filler-rubber network was generated by UV initiated thiol-ene reactions between $\mathrm{m}-\mathrm{CNCs}$ and NR molecules. Compared with a UV induced thiol-ene reaction, the heat induced thiol-ene click reaction was not affected by the light transmittance of the materials, making it easier to regulate the structure and properties of the materials. In fact, rubber composites must undergo a crosslinking process at high temperature to achieve optimal performance. This process creates the prerequisites for a thiol-ene click reaction triggered by heat. Therefore, a heat induced thiol-ene click reaction is more applicable to rubber composites.

In this research, the functionalized GO/SBR composites with high gas barrier property were fabricated by a synergetic strategy of latex compounding method and thiol-ene click reaction. The preparation process is shown in Fig. 1 (a). GO was firstly introduced into SBR by the latex compounding method to improve the dispersion of GO in SBR. Then the resultant GO/SBR masterbatches were modified by $\gamma$-mercaptopropyltriethoxysilane (MPS - its structure is shown in Fig. 1 (b)). In this process, the MPS was in situ grafted onto GO sheets by dehydration and a MPS functionalized GO (MGO)/SBR masterbatch was obtained. In the next crosslinking process at high temperature, that followed the thiol-ene click reaction between the mercapto groups in MGO and vinyl groups in SBR molecules was triggered by heat. The chemical cross-links at the interface were formed by a thiol-ene click reaction. This unique filler-rubber network structure endows an enhanced mechanical and gas barrier property to the MGO/SBR composites relative to the comparing GO/SBR composites.

\section{Experimental section}

\subsection{Materials}

SBR latex (1502) was purchased from Jinlin Petrochemical Co., Ltd (China). GO was synthesized from natural graphite in the laboratory by a modified Hummers method [20]. $\gamma$-Mercaptopropyltriethoxysilane (MPS) was provided by Nanjing Shuguang Chemical Group co. LTD (China). Concentrated sulfuric acid $\left(\mathrm{H}_{2} \mathrm{SO}_{4}\right)$ was purchased from Beijing Chemical Works (China). Zinc oxide (ZnO), Stearic acid (SA), N-(1-methylethyl)-N'-phenyl (4010NA), N-Cyclohexyl-2-benzothiazolesulfenamide (CZ) and sulfur were all commercially available and used without any treatment.

\subsection{Preparation of $G O / S B R$ and $M G O / S B R$ composites}

Fig. 1 (a) shows the fabrication process for the SBR composites with two different crosslinked networks. Firstly, GO suspension (0.3 wt\%) was obtained by dispersing GO slurry in deionized water (DW) under ultrasound. A controlled amount of GO suspension was mixed with SBR latex by agitation for $15 \mathrm{~min}$. Next, the mixture was flocculated by $\mathrm{H}_{2} \mathrm{SO}_{4}$ solution (1 $\mathrm{wt} \%$ ). The flocculated mixture was washed several times by DW until neutral to obtain the GO/SBR masterbatch. Then the resultant GO/SBR masterbatch was cut into small pieces and put into a three-necked flask.

A specific amount of MPS, at a fixed MPS: GO mass ratio of 2.5:1, was added to a DW-ethanol mixture of a volume ratio of 1:9. Then the mixture was treated for $1 \mathrm{~h}$ using a bath sonicator. During this process, the ethoxy groups on the MPS underwent hydrolysis to form highly reactive silanol groups. Next the MPS solution was poured into the threenecked flask equipped with a reflux facility. After reaction at $78{ }^{\circ} \mathrm{C}$ for $10 \mathrm{~h}$, the mixture was filtered and washed by a sufficient amount of ethanol to remove the unreacted MPS. Then, the solids were fully dried under vacuum at $60{ }^{\circ} \mathrm{C}$ to obtain MGO/SBR masterbatches. Also, the $\mathrm{GO} / \mathrm{SBR}$ masterbatches were dried through the same procedure.

Afterwards, the GO/SBR and MGO/SBR masterbatches were

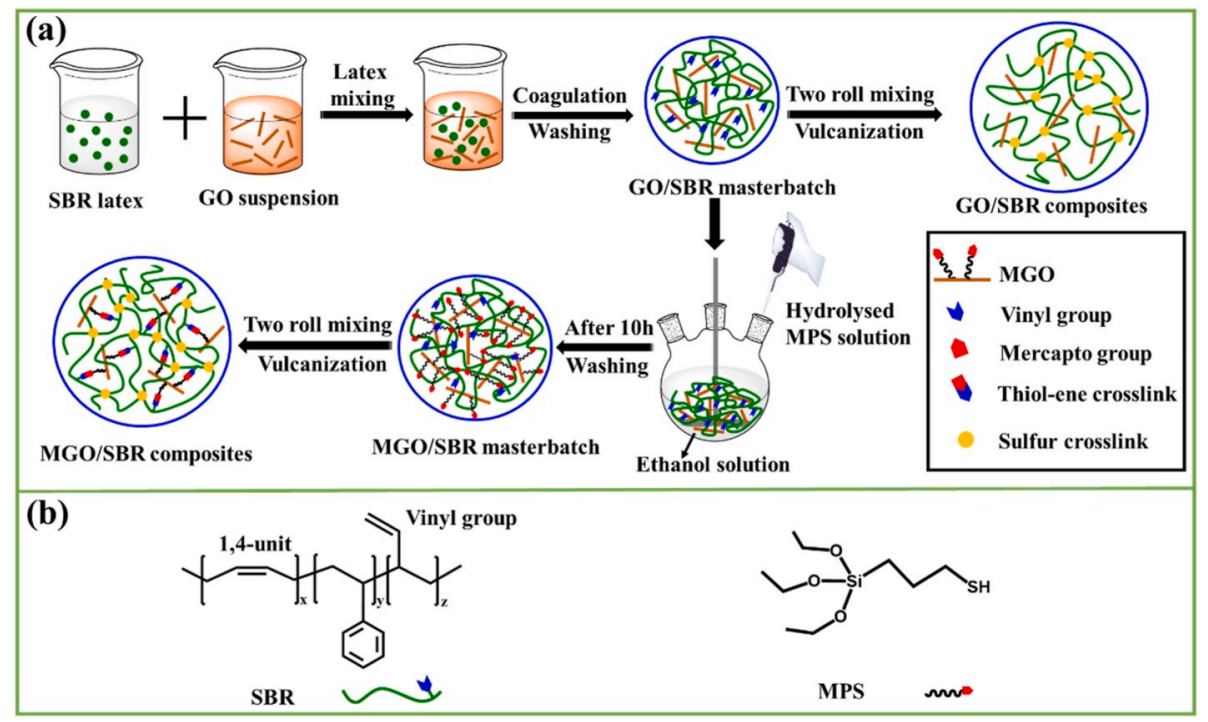

Fig. 1. (a) Schematic of the fabrication process of GO/SBR and MGO/SBR composites; (b) The chemical structure of SBR and MPS molecules. 
compounded with 5 parts per hundred rubber (phr) ZnO, 1 phr SA, 1 phr 4010NA, $1.4 \mathrm{phr}$ CZ and $2.8 \mathrm{phr}$ sulfur on a two-roll mill to obtain the GO/SBR and MGO/SBR compounds. Finally, GO/SBR and MGO/SBR composites were obtained after curing at $150{ }^{\circ} \mathrm{C}$ and $15 \mathrm{MPa}$. For simplicity, GO/SBR and MGO/SBR composites with different GO or MGO contents were named as GO/SBR-x and MGO/SBR-x, in which the $\mathrm{x}$ represents the content of GO in $100 \mathrm{phr}$ of the SBR matrix. For example, GO/SBR-1 denotes that 1 phr GO was contained in the composites.

\subsection{Preparation of $M G O$}

In order to verify the reactivity of GO with MPS, MPS modified GO (MGO) was prepared. The specific steps were as follows: the hydrolyzed MPS DW/ethanol solution was added into a GO suspension (the mass ratio of MPS:GO was 2.5:1), then the mixed solution was stirred for $10 \mathrm{~h}$ at $78{ }^{\circ} \mathrm{C}$ equipped with a reflux facility. After the reaction, the products obtained by filtering the mixture were extracted for $24 \mathrm{~h}$ in absolute ethanol. Subsequently, the extracted products were fully dried under vacuum at $60^{\circ} \mathrm{C}$. Finally, the MGO powder was obtained and used to characterize its chemical structure.

\subsection{Characterization}

Atomic force microscopy (AFM, Bruker Corporation, USA) was used to characterize the geometric parameters of GO. Fourier transform infrared (FTIR; Bruker Tensor 27), X-ray photoelectron spectroscopy analysis (XPS; ESCALAB 250), X-ray diffraction (XRD; D/Max 2500 $\mathrm{VB} 2+/ \mathrm{PC}$ ), and Raman spectroscopy (Renishaw inVia confocal) were conducted to characterize the chemical structure of MGO. Transmission electron microscope (TEM; Tecnai G2 20 S-TWIN) was used to observe the dispersion of filler in SBR. Rubber Processing Analyzer analysis (RPA; RPA 2000, Alpha Technologies) was performed to investigate the filler networks and dynamic performance of SBR composites. Curing test was performed on an oscillating disc rheometer (Model MR-C3). The mechanical test was performed on a tensile test machine (CTM 4104, SANS) according to the ISO 37: 2005 standard. The nitrogen permeability test was carried out according to the method reported by Wu et al. [21].

\section{Results and discussion}

\subsection{The chemical reaction between GO and MPS}

Since the geometric parameters (thickness and size) of GO have an important influence on the performance of rubber composites, AFM was used to characterize the geometric parameters of GO. As shown in Fig. 2 (a), it is clearly to see the lamellar structure of GO. After counting fifty GO sheets, the average thickness and size distributions of GO were obtained. The thickness of more than $98 \%$ of GO sheets is between $0.92 \mathrm{~nm}$ and $1.27 \mathrm{~nm}$, and the average thickness is $1.06 \mathrm{~nm}$, indicating that most GO were fully exfoliated and dispersed in the DW in the form of single layer. In addition, the size of GO ranges from $550 \mathrm{~nm}$ to $1.2 \mu \mathrm{m}$. To confirm the chemical reaction between GO and MPS, FTIR and XPS measurements of GO and MGO were conducted. Fig. 2 (b) shows the FTIR spectra of GO, MGO, and MPS. The characteristic peaks of $-\mathrm{OH}$ $\left(3342 \mathrm{~cm}^{-1}\right), \mathrm{C}=\mathrm{O}\left(1736 \mathrm{~cm}^{-1}\right), \mathrm{C}=\mathrm{C}\left(1628 \mathrm{~cm}^{-1}\right)$ and $\mathrm{C}-\mathrm{OH}(1401$ $\mathrm{cm}^{-1}$ ) were detected in GO [22]. The existence of these groups on GO sheets makes it easy to be functionalized, thereby allowing the tailoring the surface properties of GO. After the modification of GO by MPS, MGO exhibited two new absorption peaks for $\mathrm{Si}-\mathrm{C}\left(1236 \mathrm{~cm}^{-1}\right)$ and $\mathrm{Si}-\mathrm{O}$ $\left(1104 \mathrm{~cm}^{-1}\right)$ vibrations [23], suggesting the successful introduction of MPS onto GO sheets.

The XPS C1s spectra of GO and MGO are presented in Fig. 2 (c) and (d). There are 4 fitted peaks corresponding to $\mathrm{C}-\mathrm{C} / \mathrm{C}=\mathrm{C}, \mathrm{C}-\mathrm{O}, \mathrm{C}=\mathrm{O}$ and $\mathrm{O}-\mathrm{C}=\mathrm{O}$ groups on $\mathrm{GO}$ sheets [24]. Additionally, the peak intensity of $\mathrm{C}-\mathrm{O}$ group in MGO clearly decreased compared with GO, indicating the content of $-\mathrm{OH}$ in MGO was reduced. This is because MPS mainly reacted with $-\mathrm{OH}$ on the surface of GO. In the solution mixture of ethanol/DW, the ethoxy group of MPS underwent hydrolysis to form highly reactive silanol groups. The silanol groups dehydrated with the hydroxyl group on GO sheets, thereby chemically grafting onto its surface.

Fig. 3 (a) is the XRD patterns of GO and MGO. It's observed that a (a)
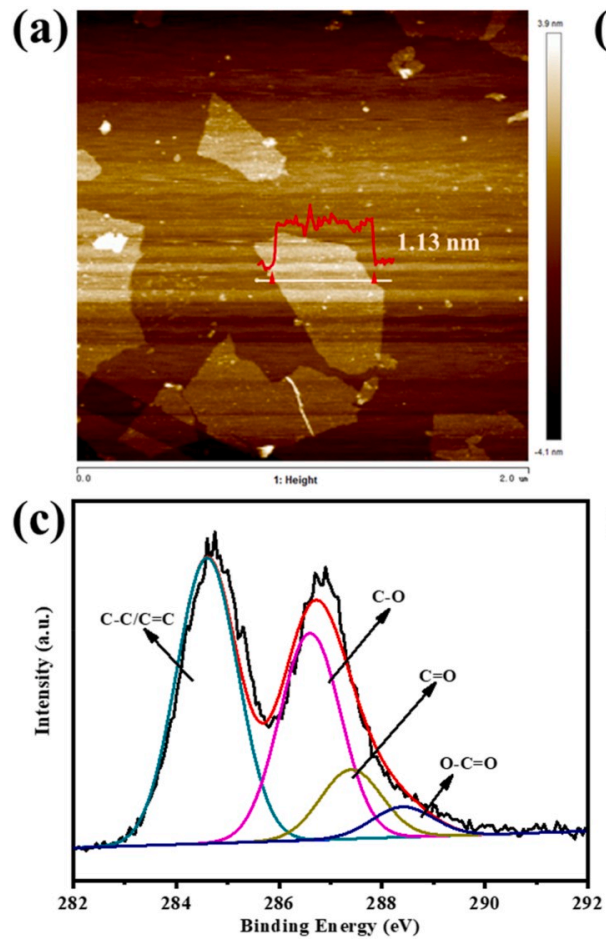

(b)
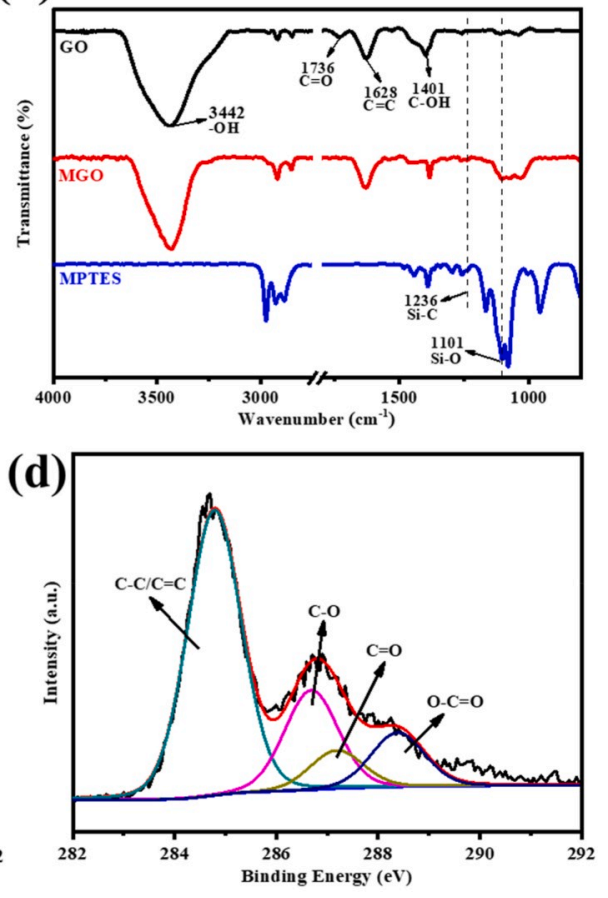

Fig. 2. (a) AFM image of GO; (b) FTIR spectra of GO, MGO, and MPS; XPS C1s spectra of (c) GO and (d) MGO. 
sharp and narrow diffraction peak of (002) plane of GO sheet at $2 \theta=$ $11.9^{\circ}$ (d-spacing is $7.43 \AA$ ). After modification, the diffraction peak at $11.9^{\circ}$ disappeared and a broad diffraction peak appeared around $20.3^{\circ}$ (d-spacing is $4.37 \AA$ ), suggesting that GO was partially reduced and some defects in the disordered stacking of MGO were introduced after grafting MPS molecules on the GO sheets [25]. The Raman spectra of GO and MGO are presented in Fig. 3 (b). As can be seen, GO and MGO both show two strong characteristic peaks of D band $\left(1350 \mathrm{~cm}^{-1}\right)$ and $\mathrm{G}$ band $\left(1590 \mathrm{~cm}^{-1}\right)$. The intensity of $\mathrm{D}$ band and $\mathrm{G}$ band increased from 0.89 to 1.06 for GO and MGO, indicating the disorder degree increased after the grafting of MPS on the GO sheet [26]. Upon the above analysis, it is proved that MPS was grafted to GO surfaces in the DW/ethanol system. The reaction mechanism between MPS and GO is shown in Fig. 4 (a).

\subsection{The thiol-ene click reaction between MGO and SBR molecules}

During the crosslinking process at high temperature, thiol-ene reaction triggered by heat took place between mercapto group in MPS and $\mathrm{C}=\mathrm{C}$ group of SBR. The mercapto groups preferentially reacted with $\mathrm{C}=\mathrm{C}$ in vinyl groups rather than in $1,4-\mathrm{C}=\mathrm{C}$ groups of $\mathrm{SBR}$ molecules due to the bigger space hindrance of the $1,4-\mathrm{C}=\mathrm{C}$ groups in the main chains [27]. In order to verify the thiol-ene click reaction between mercapto group in MPS and vinyl group in SBR molecules, MPS was directly added to the SBR matrix by mechanical mixing and no other additives were added in the resultant MPS/SBR compound. After the MPS/SBR compound was heat-treated at $150{ }^{\circ} \mathrm{C}$, an ATR-FTIR test was performed on the compound before and after heat treatment, as presented in Fig. 5 (a). There are two peaks at 912 and $699 \mathrm{~cm}^{-1}$ corresponding to the vibration of the vinyl group and styrene ring, respectively [28]. Since the structure and content of styrene didn't change during the heat treatment, the peak at $699 \mathrm{~cm}^{-1}$ was selected as the internal standard for normalization to determine the content of vinyl groups in the MPS/SBR compound before and after heat treatment [29]. The absorbance at $912 \mathrm{~cm}^{-1}$ decreased from 0.59 for untreated the MPS/SBR compound to 0.47 for the SBR/MPS compound after heat treatment, suggesting that part of the vinyl groups in SBR were consumed by MPS during the thiol-ene click reaction.

To further verify the thiol-ene click reaction between the mercapto group in MPS and the vinyl group in SBR molecules, curing tests were performed at $150{ }^{\circ} \mathrm{C}$ on different SBR compounds and the resulting curves are presented in Fig. 5 (b). The measured torque for pure SBR was basically unchanged over time. In contrast, the torque of the MPS/SBR compound changed minimally within $10 \mathrm{~min}$ and then increased slightly over $10 \mathrm{~min}$, indicating the thiol-ene reaction between MPS and SBR occurred and a weak three-dimensional crosslinking network had formed after $10 \mathrm{~min}$. In fact, MPS molecules just dangled in the SBR molecules via thiol-ene reaction, thus the torque was constant within 10 min for SBR/MPS compounds. In this process, the ethoxy groups in MPS pyrolyzed and generated highly active silanols. Under continuous heating, silanols polymerized with each other and formed a weak three- dimensional crosslinked network, resulting the increased torque. In addition, the torque exhibited by the GO/SBR- 5 masterbatch was almost constant over the time. In contrast, after MPS was in situ grafted onto the surface of GO, the torque capacity of MGO/SBR-5 masterbatch increased significantly over the time, suggesting a strong three-dimensional crosslinking network was formed. After modification, the MGO could act as a crosslinking center, thus SBR molecules could be crosslinked by MGO through the thiol-ene reaction (the reaction mechanism is shown in Fig. 4 (b)).

Therefore, the mercapto groups in MGO participated in the crosslinking of rubber molecules and formed a GO-MPS-rubber network at interface in the MGO/SBR composites, in addition to the crosslinking network formed by sulfur. The construction of this unique network results in the strong chemical interactions between MGO and SBR molecules.

\subsection{The dispersion state of $M G O$ and the filler networks in $S B R$ composites}

TEM images can be used to visually observe the dispersion of the fillers in the matrix. As shown in Fig. 6, the dark gray lines in the image represent GO or MGO and the light gray area represents the SBR matrix. In Fig. 6 (a), the regions of SBR matrix shown as light gray were exposed and not fully covered by GO. In contrast, most of the SBR matrix was covered by MGO as shown in Fig. 6 (b). For equal filler contents, the larger the area of rubber matrix covered by fillers, the better the dispersibility of the fillers. Therefore, the dispersion of MGO is more uniform than that of GO in the SBR matrix. Although the latex compounding could ensure the uniform dispersion of GO in SBR, some GO aggregations were inevitable during the crosslinking process [30]. After modification, the hydrophobicity of MGO increased, thus its compatibility with the SBR matrix was enhanced. Meanwhile, the chemical interfaces between MGO and SBR molecules were also beneficial for improving the dispersion of MGO.

To investigate the filler network constructed by GO or MGO in the SBR matrix, RPA analysis was used. In Fig. 7 (a), the shear storage modulus (G') of the GO/SBR-5 compound was significantly larger than that of the pure SBR compound, indicating strong filler networks were constructed by GO sheets in SBR. As for MGO/SBR- 5 compound, the value of G' was further increased by comparison with the GO/SBR-5 compound. This improvement was mainly ascribed to the improved compatibility between MGO and SBR molecules; thus SBR molecules were bridged by the neighboring MGO sheets, thereby forming a tight filler network. The formation of such a tight filler network would be in favor of forming tortuous paths thus prolonging the infiltration time of small gas molecules. Although the strong filler network will result in high energy dissipation which is not desirable for tire materials, the uniform dispersion of fillers and strong interactions between fillers and SBR molecules will weaken the internal friction, thereby reducing the energy dissipation. As shown in Fig. 7 (b), the G' of MGO/SBR-5
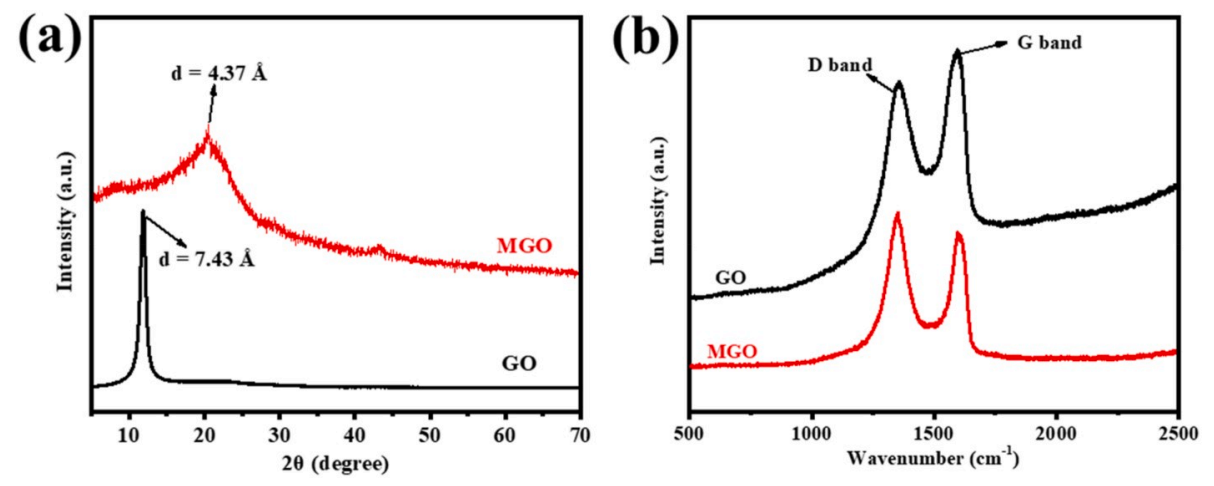

Fig. 3. (a) XRD and (b) Raman spectra of GO and MGO. 


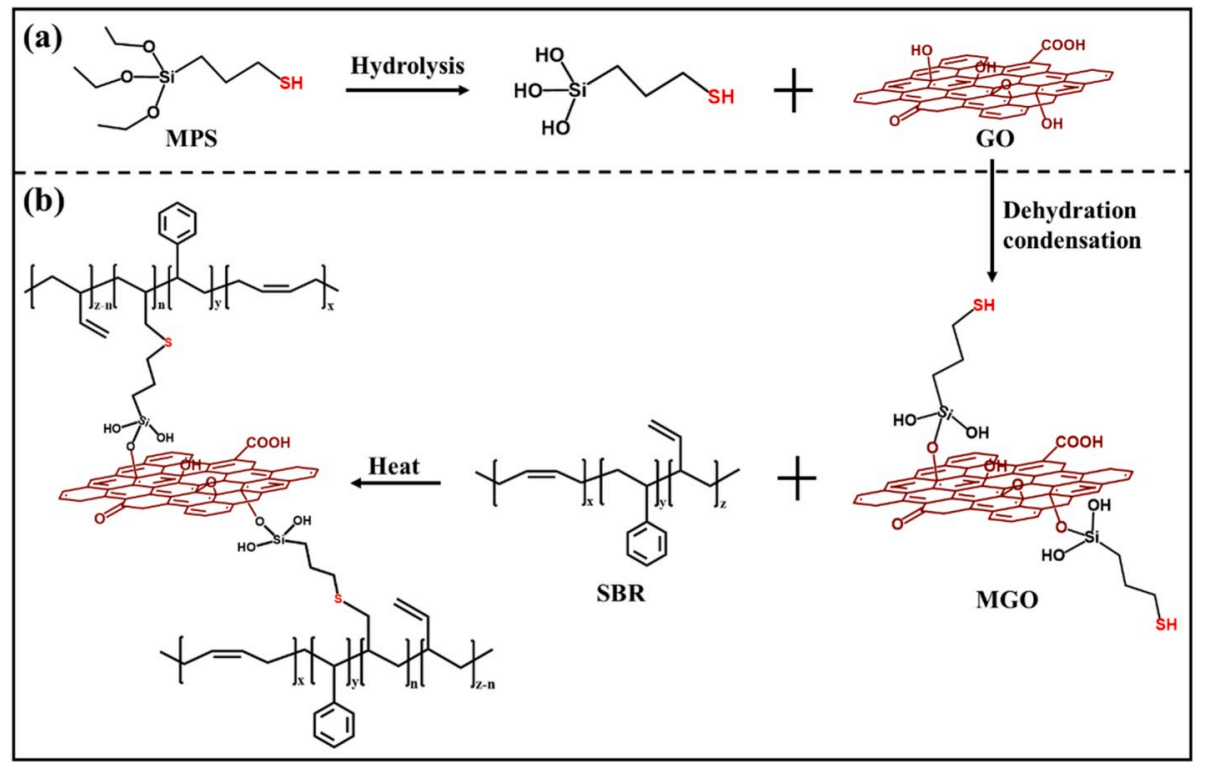

Fig. 4. Reaction formula of (a) GO and MPS by dehydration condensation, and of (b) SBR and MGO triggered by heat.
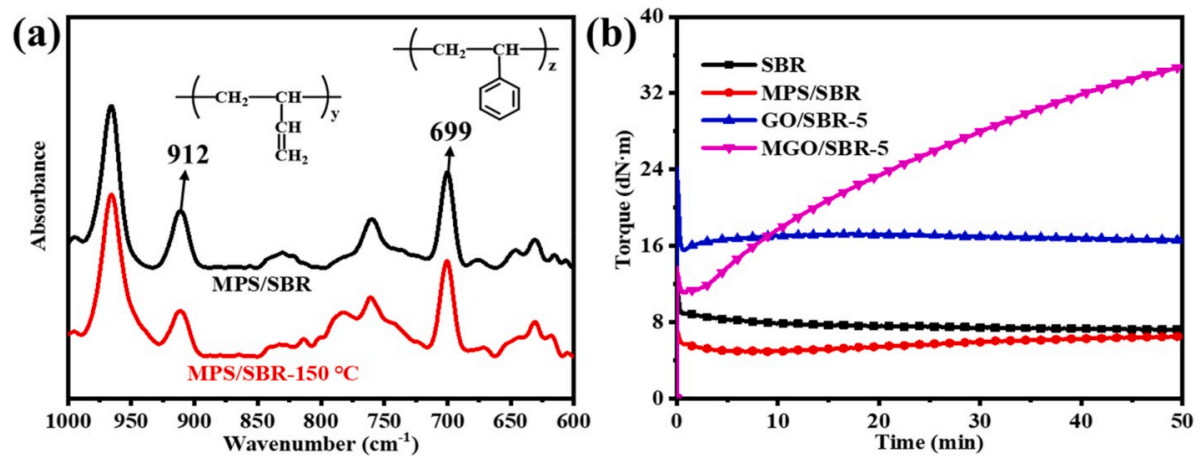

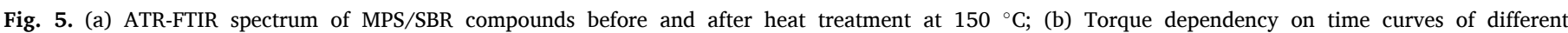
SBR compounds.
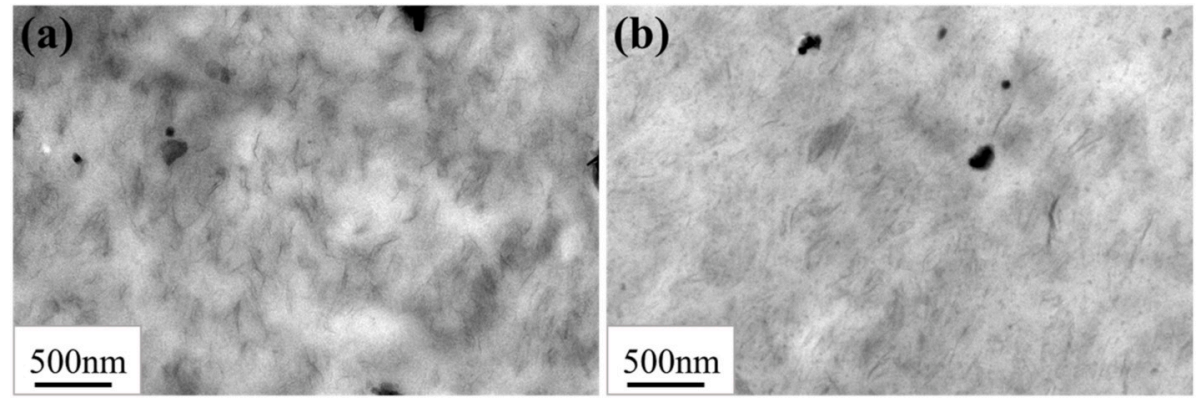

Fig. 6. TEM images of SBR composites filled with $5 \mathrm{phr}$ (a) GO and (b) MGO.

composite is larger than that of the GO/SBR-5 composite, also reflecting the stronger interactions between MGO and SBR molecules. Meanwhile, as seen in Fig. 7 (c), the loss factor ( $\tan \delta$ ) of the MGO/SBR- 5 composite is lower than that of the GO/SBR-5 composite when the applied strain under $20 \%$. In particular, the value of $\tan \delta$ of MGO/SBR composites reduces by $14.8 \%$ compared to that of GO/SBR composites when the strain is $7 \%$, suggesting the MGO/SBR composites have lower rolling resistance and energy dissipation.

\subsection{Crosslinking characterization of $M G O / S B R$ composites}

Fig. 8 shows the crosslinking characteristic of SBR compounds. In Fig. 8 (a), the scorch time (T10) and optimum curing time (T90) increased with increases in the GO content. The delay of the overall crosslinking time was caused by the acidic oxygen groups on GO sheet absorbing the curing agents [31]. After the modification by MPS, the T10 and T90 of MGO/SBR compounds significantly reduced compared to those of GO/SBR compounds for the same filler content. This was because some of the oxygen groups were consumed when MPS was 

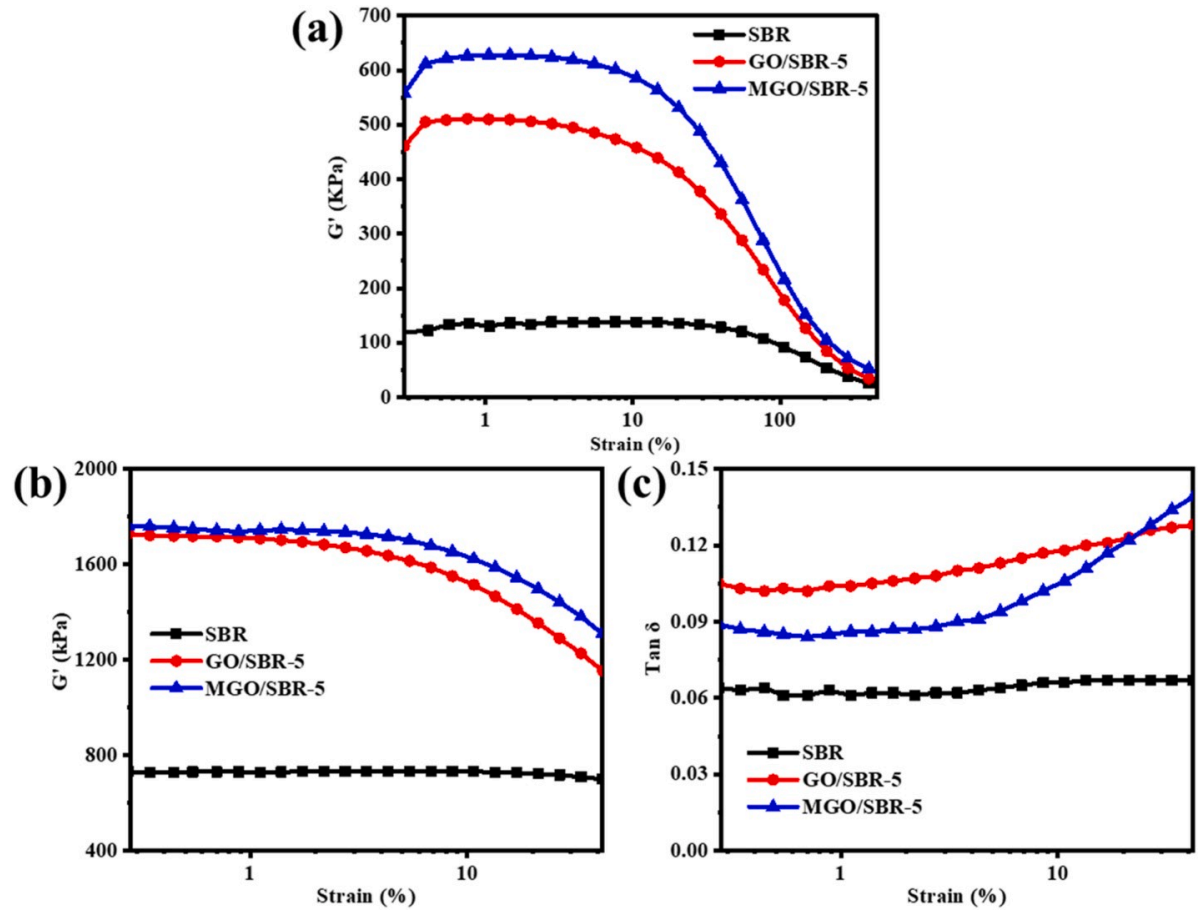

Fig. 7. (a) Shear storage modulus versus dynamic strain amplitudes of SBR, GO/SBR-5 and MGO/SBR-5 compounds; (b) Shear storage modulus and (c) loss factor versus dynamic strain amplitude of SBR, GO/SBR-5 and MGO/SBR-5 composites.
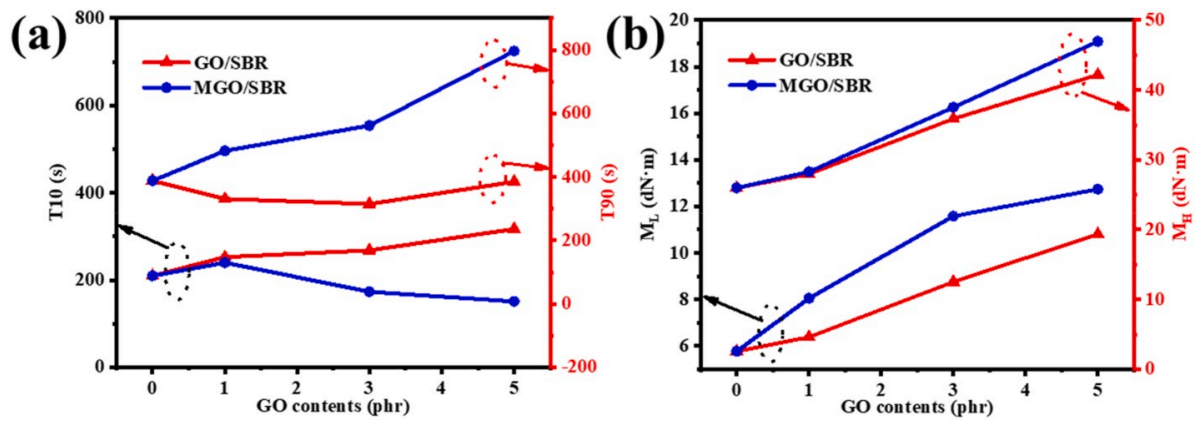

Fig. 8. (a) T10 and T90 versus GO content, and (b) minimum torque and maximum torque values of GO/SBR and MGO/SBR composites versus GO content.

grafted onto GO sheets. Meanwhile, the mercapto groups participated in the crosslinking reaction, thus the crosslinking process was promoted. In this process, the thiol-ene reaction induced the formation of a crosslinked network at the interface between MGO and SBR molecules in addition to the crosslinking network formed by sulfur. Fig. 9 shows the diagram of the difference of the crosslinked networks between GO/SBR composites and $\mathrm{MGO} / \mathrm{SBR}$ composites. In rubber composites, the maximum torque $\left(\mathrm{M}_{\mathrm{H}}\right)$ and minimum torque $\left(\mathrm{M}_{\mathrm{L}}\right)$ are mainly determined by the filler networks and interface strength. In Fig. 8 (b), the values of $\mathrm{M}_{\mathrm{L}}$ and $\mathrm{M}_{\mathrm{H}}$ were higher in MGO/SBR compounds than those in

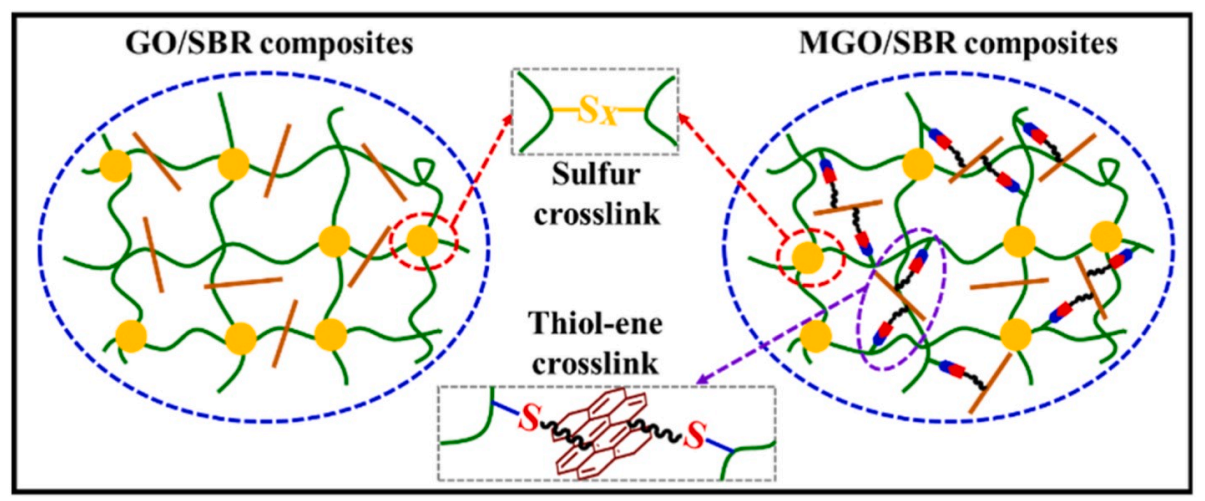

Fig. 9. A diagrammatic representation of the single crosslinked network in GO/SBR composites and double crosslinked networks in MGO/SBR composites. 
GO/SBR compounds, demonstrating the filler networks and interface interactions between MGO and SBR molecules were stronger in MGO/SBR

\subsection{Mechanical properties of MGO/SBR composites}

Fig. 10 (a) shows the strain-stress curves of all SBR composites, and the values of tensile strength, modulus at $300 \%$ strain and elongation at break are presented in Fig. 10 (b). The tensile strength of GO/SBR and MGO/SBR composites gradually increased with the increase in GO and MGO content. Meanwhile, the enhancement effect of MGO on SBR is more significant than that of GO on SBR at the same filler content. For example, when filling $5 \mathrm{phr}$ fillers, the tensile strength of the MGO/SBR composite improved $19.0 \%$ relative to that of the GO/SBR composite. The higher modulus also reflected the reinforcing effect of the filler on the SBR composites. In Fig. 10 (b), when filling the same amount of filler content, the modulus at $300 \%$ strain of the MGO/SBR composite is obviously larger than that of the GO/SBR composite. When the filler content is up to $5 \mathrm{phr}$, the modulus at 300\% strain of MGO/SBR composite improved by $64.9 \%$ when compared with that of the GO/SBR composite. Such an excellent reinforcing effect of the MGO on SBR composites is mainly stemming from the uniform dispersion of MGO and strong chemical interactions in SBR. In addition, the elongation at break for MGO/SBR composites decreased when compared with that for the GO/SBR composites at the same filler contents, as shown in Fig. 10 (b). This phenomenon is also ascribed from the strong interactions between MGO and SBR molecules, which restricted the movement of SBR molecules when stretching the composites.

In order to further evaluate the interfaces between GO or MGO and SBR molecules, Mooney-Rivlin curves were obtained by converting the stress-strain curves according to Mooney-Rivlin equation (see equation (1)) [32].

$\sigma^{*}=\frac{\sigma}{\lambda-\lambda^{-2}}=2 C_{1}+2 C_{2} / \lambda$

In equation (1), $\sigma$ is the stress, $\lambda$ is the extension ratio and $C_{1}$ and $C_{2}$ are constants that are independent of $\lambda$. The plots of reduced stress $\left(\sigma^{*}\right)$ against the reciprocal of $\lambda$ for all SBR composites are shown in Fig. 10 (c). When filling $1 \mathrm{phr}$ filler, the $\sigma^{*}$ of GO/SBR and MGO/SBR composites first decreased and then reached a plateau as the strain increased $\left(\lambda^{-1}\right.$ decreased). As the strain further increased, a point of inflection upwards appears in the curve. When the filler content exceeds $3 \mathrm{phr}$, the plateau disappears and the upturn occurs at a smaller strain. The inflection points were owing to the rubber molecules adsorbing on the GO or MGO sheets, which restricted the movement of rubber molecules. During the stretching process, the rubber molecules were gradually straightened and strain hardened, resulting in a sharp increase in $\sigma^{*}$. As shown in Fig. 10 (c), the upturn occurs at smaller deformations for MGO/SBR composites than that for GO/SBR composites. This phenomenon was ascribed to the MGO serving as the crosslinking center and forming strong chemical interactions.

\subsection{Gas barrier property of $M G O / S B R$ composites}

Nitrogen permeability (P) for all SBR composites is presented in Fig. 11 (a). With the increase in filler contents, the $P$ values for GO/SBR and MGO/SBR composites decreased. Differently, the $\mathrm{P}$ values of MGO/ SBR composites are much lower relative to those comparing GO/SBR composites at the same filler contents, demonstrating that MGO/SBR composites have better gas barrier performance than GO/SBR composites. When the content of GO or MGO was $5 \mathrm{phr}$, the P value of the MGO/ SBR composite decreased by $36.8 \%$ relative to the comparing GO/SBR composite. In general, the effect of lamellar filler on the gas barrier property of rubber materials is largely determined by the interface strength and dispersion state of fillers in rubber composites [33]. After the modification by MPS, MGO had improved dispersibility in SBR and heightened interface adhesion with SBR molecules; thus the pathways and diffusion times were prolonged when the small gas molecules permeating through the SBR matrices. Moreover, the double crosslinked networks in MGO/SBR composites resulted in the reduction of free volumes among SBR molecules, further decreasing the penetration of small gas molecules.

In practical applications, especially in tires, both the barrier properties and modulus at $300 \%$ strain of the rubber composites are important factors that need to be focused on. In order to access the progressivity of the MGO/SBR composites in both barrier properties and modulus at $300 \%$ strain, the previously reported barrier properties and
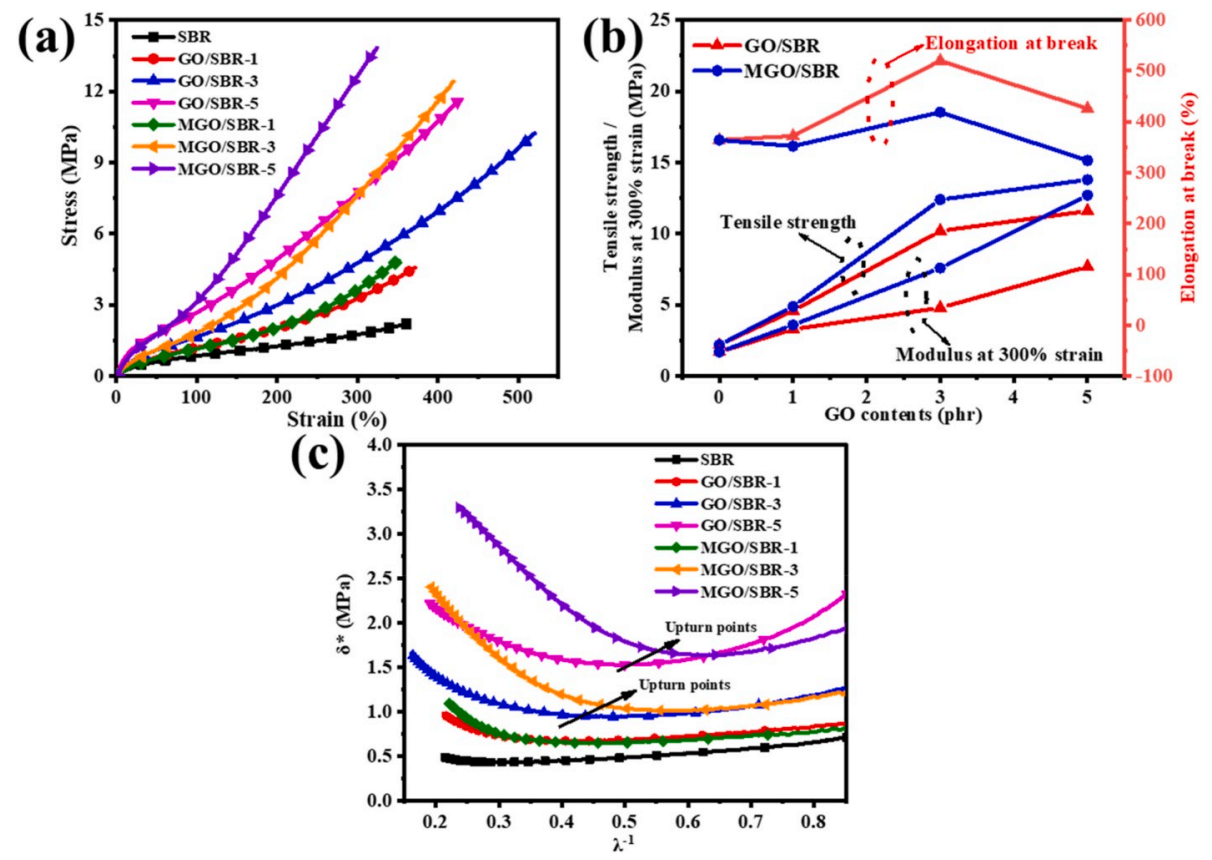

Fig. 10. (a) The strain-stress curves of all SBR composites; (b) The values of tensile strength, modulus at $300 \%$ strain and elongation at break of all SBR composites; (c) Mooney-Rivlin curves of all SBR composites. 

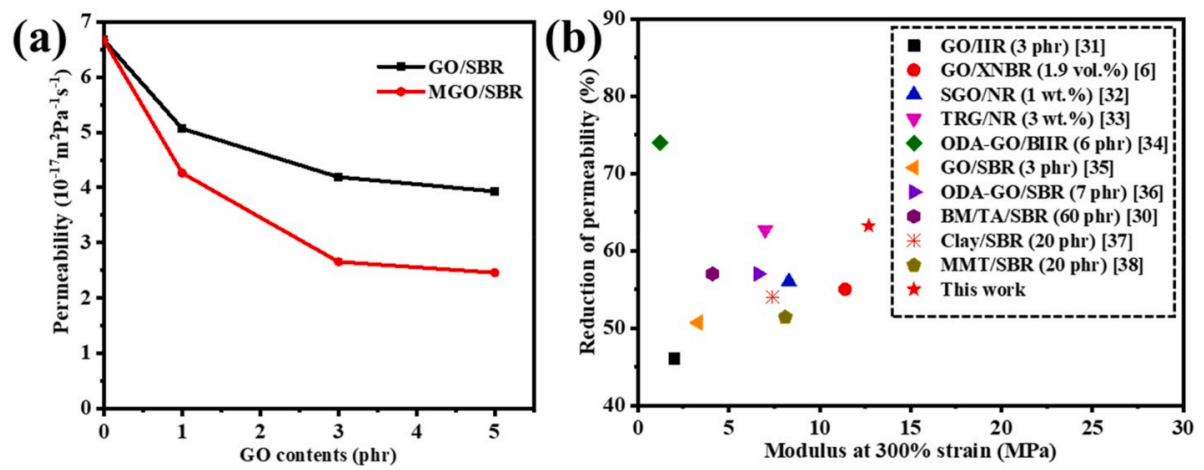

Fig. 11. (a) Nitrogen permeability for all SBR composites; (b) Comparison of modulus at $300 \%$ strain and reduction of permeability between this work and other previously published works. Data in the bracket is the filler content.

modulus at $300 \%$ strain for lamellar fillers filled rubber composites with uniform filler networks [8,33-41] are summarized in Fig. 11 (b). Considering that the rubber matrix and fillers are different in different works, the permeability reduction of the filled rubber composites relative to the pure rubber matrix was compared. By comparison, the MGO/SBR composites with double crosslinked networks exhibit comprehensive advantage in the improvement of both modulus at $300 \%$ strain and barrier properties.

\section{Conclusions}

The GO/SBR masterbatch was fabricated by the latex compounding method. Subsequently, MPS was in situ grafted onto GO sheets to obtain the MGO/SBR masterbatch. In the subsequent crosslinking process at high temperature, MGO was chemically coupled with SBR molecules via a thiol-ene click reaction. The resultant MGO/SBR composites with strong chemical filler-rubber networks and double crosslinked networks possessed higher mechanical and gas barrier properties. When filling 5 phr fillers, the tensile strength, modulus at $300 \%$ strain and gas barrier properties of the MGO/SBR improved $19.0 \%, 64.9 \%$ and $37.5 \%$, respectively, relative to the comparing GO/SBR composite.

\section{Declaration of competing interest}

The authors declare that they have no known competing financial interests or personal relationships that could have appeared to influence the work reported in this paper.

\section{CRediT authorship contribution statement}

Long Zheng: Conceptualization, Methodology, Investigation, Data curation, Writing - original draft, Visualization. Stephen Jerrams: Writing - review \& editing. Tian Su: Conceptualization, Methodology, Investigation, Data curation. Zongchao Xu: Methodology, Investigation. Liqun Zhang: Supervision, Investigation. Li Liu: Methodology, Supervision, Investigation. Shipeng Wen: Conceptualization, Investigation, Supervision, Writing - review \& editing.

\section{Acknowledgments}

The authors gratefully appreciate the financial support from the National Key R\&D Program of China (2017YFE0126800), and the National Natural Science Foundation of China (51573007).

\section{References}

[1] Amato F, Cassee FR, Denier van der Gon HA, Gehrig R, Gustafsson M, Hafner W, et al. Urban air quality: the challenge of traffic non-exhaust emissions. J Hazard Mater 2014;275:31-6.
[2] Hou G, Tao W, Liu J, Zhang X, Dong M, Zhang L. Effect of the structural characteristics of solution styrene-butadiene rubber on the properties of rubber composites. J Appl Polym Sci 2017;135(24):45749.

[3] Nikiel L, Gerspacher M, Yang H, O'Farrell CP. Filler dispersion, network density, and tire rolling resistance. Rubber chemistry and technology 2001;74(2):249-59.

[4] Cao L, Sinha TK, Tao L, Li H, Zong C, Kim JK. Synergistic reinforcement of silanized silica-graphene oxide hybrid in natural rubber for tire-tread fabrication: a latex based facile approach. Compos B Eng 2019;161:667-76.

[5] Waddell Walter H, Napier R Christopher, Evans Larry R, Popio James A, Douglas Rice, Rajapakshe M. How tread loss affects rolling resistance. Rubber Plast News 2014;(May 5):14-8.

[6] Zhang Q, Xu J. Application of clay/SBR nanocomposite in the inner liner of semisteel radial Tire. Rubber Science and Technology 2015;(7):43-6.

[7] He SJ, Zhang LQ, Wu XH, Lin J, Xi MM, Wang YQ. Reduction of the filler network interaction in novel inner liner compound based on SBR/rectorite nanocomposite by glycerin. Polym Compos 2012;33(3):336-42.

[8] Kang H, Zuo K, Wang Z, Zhang L, Liu L, Guo B. Using a green method to develop graphene oxide/elastomers nanocomposites with combination of high barrier and mechanical performance. Compos Sci Technol 2014;92:1-8.

[9] Sadasivuni KK, Saiter A, Gautier N, Thomas S, Grohens Y. Effect of molecular interactions on the performance of poly(isobutylene-co-isoprene)/graphene and clay nanocomposites. Colloid Polym Sci 2013;291(7):1729-40.

[10] Tang Z, Zhang L, Feng W, Guo B, Liu F, Jia D. Rational design of graphene surface chemistry for high-performance rubber/graphene composites. Macromolecules 2014;47(24):8663-73.

[11] Chen L, Jia Z, Guo X, Zhong B, Chen Y, Luo Y, et al. Functionalized HNTs nanocluster vulcanized natural rubber with high filler-rubber interaction. Chem Eng J 2018;336:748-56.

[12] Liu X, Kuang W, Guo B. Preparation of rubber/graphene oxide composites with insitu interfacial design. Polymer 2015;56:553-62.

[13] Lutz J-F. 1,3-Dipolar cycloadditions of azides and alkynes: a universal ligation tool in polymer and materials science. Angew Chem Int Ed 2007;46(7):1018-25.

[14] Le Droumaguet B, Velonia K. Click chemistry: a powerful tool to create polymerbased macromolecular chimeras. Macromol Rapid Commun 2008;29(12-13): 1073-89.

[15] Hawker CJ. The convergence of synthetic organic and polymer chemistries. Science 2005;309(5738):1200-5.

[16] Uygun M, Tasdelen MA, Yagci Y. Influence of type of initiation on thiol-ene "click" chemistry. Macromol Chem Phys 2010;211(1):103-10.

[17] Lowe AB. Thiol-ene "click" reactions and recent applications in polymer and materials synthesis. Polym Chem 2010;1(1):17-36.

[18] Ma T, Li L, Wang Q, Guo C. Construction of intumescent flame retardant and hydrophobic coating on wood substrates based on thiol-ene click chemistry without photoinitiators. Compos B Eng 2019;177:107357.

[19] Parambath Kanoth B, Claudino M, Johansson M, Berglund LA, Zhou Q. Biocomposites from natural rubber: synergistic effects of functionalized cellulose nanocrystals as both reinforcing and cross-linking agents via free-radical thiol-ene chemistry. ACS Appl Mater Interfaces 2015;7(30):16303-10.

[20] Hummers WS, Offeman RE. Preparation of graphitic oxide. J Am Chem Soc 1958; 80(6):1339.

[21] Wu YP, Wang YQ, Zhang HF, Wang YZ, Yu DS, Zhang LQ, et al. Rubber-pristine clay nanocomposites prepared by co-coagulating rubber latex and clay aqueous suspension. Compos Sci Technol 2005;65(7-8):1195-202.

[22] Cui J, Xu J, Li J, Qiu H, Zheng S, Yang J. A crosslinkable graphene oxide in waterborne polyurethane anticorrosive coatings: experiments and simulation. Compos B Eng 2020;188:107889.

[23] Liu L, Tian L, Zhao G, Huang Y, Wei Q, Cao W. Ultrasensitive electrochemical immunosensor for alpha fetoprotein detection based on platinum nanoparticles anchored on cobalt oxide/graphene nanosheets for signal amplification. Anal Chim Acta 2017;986:138-44.

[24] Fang F, Ran S, Fang Z, Song P, Wang H. Improved flame resistance and thermomechanical properties of epoxy resin nanocomposites from functionalized graphene oxide via self-assembly in water. Compos B Eng 2019;165:406-16. 
[25] Wang Y, Wang W, Xu R, Zhu M, Yu D. Flexible, durable and thermal conducting thiol-modified rGO-WPU/cotton fabric for robust electromagnetic interference shielding. Chem Eng J 2019;360:817-28.

[26] Ma L, Zhu Y, Feng P, Song G, Huang Y, Liu H, et al. Reinforcing carbon fiber epoxy composites with triazine derivatives functionalized graphene oxide modified sizing agent. Compos B Eng 2019;176:107078.

[27] Sun Z, Huang Q, Wang Y, Zhang L, Wu Y. Structure and properties of silicone rubber/styrene-butadiene rubber blends with in situ interface coupling by thiolene click reaction. Ind Eng Chem Res 2017;56(6):1471-7.

[28] Bellas R, Díez J, Rico M, Barral L, Ramírez C, Montero B. Accelerated ageing of styrene-butadiene rubber nanocomposites stabilized by phenolic antioxidant. Polym Compos 2014;35(2):334-43.

[29] De Sarkar M, De PP, Bhowmick AK. Influence of hydrogenation and styrene content on the unaged and aged properties of styrene-butadiene copolymer. J Mater Sci 1999;34(8):1741-7.

[30] Wang D, Chen S, Chen L, Chen B, Ren F, Zhu C, et al. Investigation and improvement of the scorch behavior of silica-filled solution styrene-butadiene rubber compound. J Appl Polym Sci 2019;136(35):47918.

[31] Hernandez M, del Mar Bernal M, Verdejo R, Ezquerra TA, Lopez-Manchado MA. Overall performance of natural rubber/graphene nanocomposites. Compos Sci Technol 2012;73:40-6.

[32] Rivlin RS. The elasticity of rubber. Rubber Chemistry \& Technology 1992;65(3): $51-66$.

[33] Tang Z, Zhang C, Zhu L, Guo B. Low permeability styrene butadiene rubber/ boehmite nanocomposites modified with tannic acid. Mater Des 2016;103:25-31.
[34] Zheng L, Wang DY, Xu ZC, Zhang LQ, Liu L, Wen SP. High barrier properties against sulfur mustard of graphene oxide/butyl rubber composites. Compos Sci Technol 2019;170:141-7.

[35] Wu J, Huang G, Li H, Wu S, Liu Y, Zheng J. Enhanced mechanical and gas barrier properties of rubber nanocomposites with surface functionalized graphene oxide at low content. Polymer 2013;54(7):1930-7.

[36] Yaragalla S APM, Kalarikkal N, Thomas S. Chemistry associated with natural rubber-graphene nanocomposites and its effect on physical and structural properties. Ind Crop Prod 2015;74:792-802.

[37] Yang X, Zhang Y, Xu Y, Gao S, Guo S. Effect of octadecylamine modified graphene on thermal stability, mechanical properties and gas barrier properties of brominated butyl rubber. Macromol Res 2017;25(3):270-5.

[38] Zheng L, Jerrams S, Xu Z, Zhang L, Liu L, Wen S. Enhanced gas barrier properties of graphene oxide/rubber composites with strong interfaces constructed by graphene oxide and sulfur. Chem Eng J 2020;383:123100.

[39] Su T, Xie L, Zheng L, Xu Z, Liu L, Wen S. Structure and properties of octadecylamine modified graphene oxide/ESBR composite. Rubber Industry 2019; 66(2):89-96.

[40] Yang J. Rubber-pristine clay nanocomposites prepared by co-coagulating rubber latex and clay aqueous suspension. Compos Sci Technol 2005;65(7-8):1195-202.

[41] Zhang H, Wang Y, Wu Y, Zhang L, Yang J. Study on flammability of montmorillonite/styrene-butadiene rubber (SBR) nanocomposites. J Appl Polym Sci 2005;97(3):844-9. 